\title{
Dibutylphthalate and Tween-80 up-regulate peroxisome numbers in Candida albicans ATCC 36232
}

\author{
Maureen Downey ${ }^{1}$, Jonathan Blaize ${ }^{1}$, William J. Lamoreaux ${ }^{1,2}$, and Elena C. McCoy ${ }^{1}$ \\ ${ }^{1}$ Department of Biology, The College of Staten Island, The City University of New York, 2800 \\ Victory Blvd., Staten Island, NY 10314 USA \\ ${ }^{2}$ Institute for Macromolecular Assemblies, The City University of New York, 2800 Victory Blvd., \\ Staten Island, NY 10314 USA
}

Certain xenobiotics can alter cell metabolism by inducing biochemical and morphological changes in target cells, including peroxisome proliferation. Peroxisome proliferation, in turn, is a candidate marker for carcinogenesis in mammalian tissues. Phthalates are of great environmental concern; since they are found in plastics. Dibutylphthalate (DBP) is a plasticizer that induces peroxisome proliferation in rat liver [1] and can elicit estrogenic responses in yeast [2]. In yeast, peroxisomes are the site of specific catabolic pathways that characteristically include hydrogen peroxide producing oxidases and catalase [3]. Polyoxyethylenesorbitane-monooleate, or Tween 80 , is a nonionic surfactant that can induce catalase function in yeast and is used in assays to characterize yeast catalase activity [4]. Although Tween 80 can affect peroxisomal catalase, it is not effectively degraded in Saccharomyces cerevisiae using the peroxisomal $\beta$-oxidation pathway [5]. For these studies, we sought to use rapidly growing cultures of yeast to test for peroxisome proliferation resulting from treatment with certain xenobiotics. Our purpose was to determine morphological changes occurring in these yeast exposed to dibutylphthalate (DBP), Tween 80, or both (DBPT). Cultures of Candida albicans 32632 were grown in yeast nutrient broth with dextrose (YND). Experimental cultures were supplemented with $0.5 \%$ DBP or $0.05 \%$ Tween 80 , or both, and cultured for 48 hours at $37^{\circ} \mathrm{C}$. Cultures were then processed for transmission electron for routine morphology or confocal laser scanning microscopy to localize catalase activity.

For the control cultures, we observed some immunofluorescence in the control cultures (Figure 1A). When examining the treated cultures, cells grown in DBP had stronger signals than all other cultures (Figure 1B), with those treated with Tween 80 intermediate to DBP and DBPT (Figure 1C, 1D). The DBPT-treated cultures were the least strongly stained of the treated cultures (Figure 1D). By EM analyses, the cytoplasm appears to have abundant ribosomes with well dispersed mitochondria located along the cell periphery and an electron dense layer on the outer cell wall (Figure 2A). DBPtreated cells exhibited cell wall peeling with an overall increase in presumed peroxisomes (Figure 2B). In cells treated with Tween 80, we observed an increased thickness in the cell wall (Figure 2C). Cells treated with DBPT did not show cell wall peeling as associated with DBP alone, but the cell wall thickness was greater than in the DBP-treated cells (Figure 2D). The data strongly suggest that C. albicans can interact with estrogenic responsive chemicals, such as phthalates and long-chain fatty acids found in oleates like Tween 80 .

[1] W. Kaufmann, et al., Regul Toxicol Pharmacol (2002)], 36.

[2] H. R. Andersen, et al., Environ Health Perspect (1999)], 107 Suppl 1.

[3] M. Veenhuis, F. A. Salomons, I. J. Van Der Klei, Microsc Res Tech (2000)], 51.

[4] P. Zimniak, et al., Eur J Biochem (1976)], 71.

[5] A. Gurvitz,et al. J Biol Chem (2001)], 276. 
A
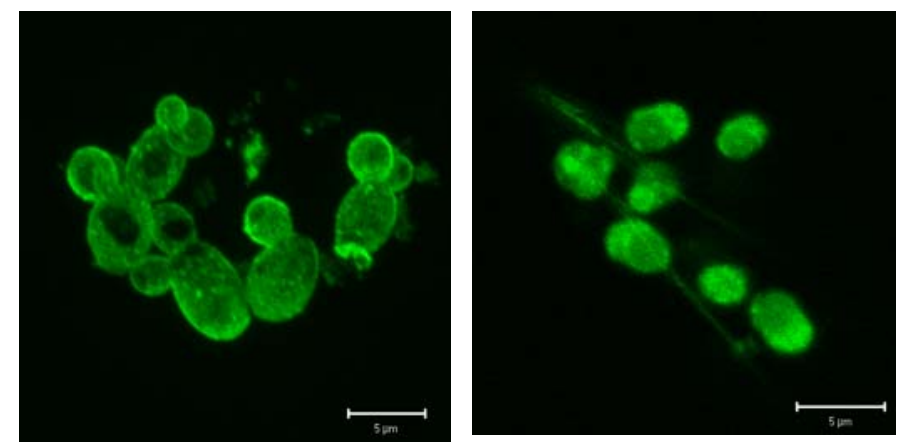

B
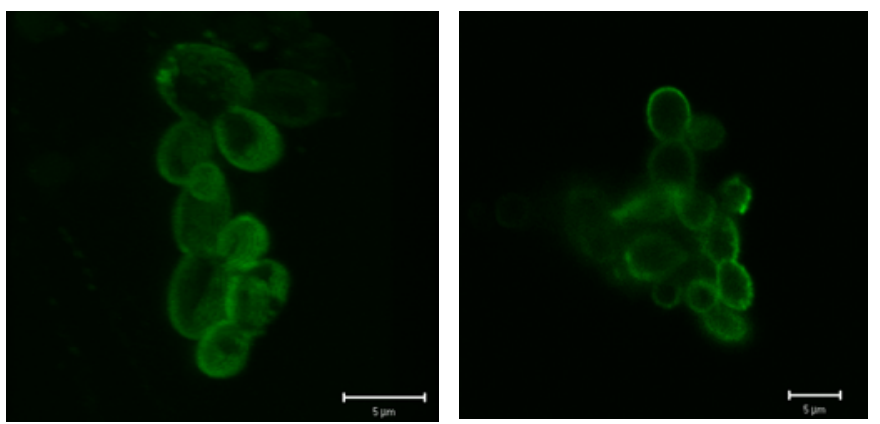

Figure 1. C. albicans 36232 catalase localization. A. Control cells express basal levels of catalase. B. Cells treated with DBP demonstrate increased catalase activity. C. In Tween-treated cells the catalase levels are less than DBP-treated and control cells. D. The DBPT-treated cells contain the least amount of catalase.
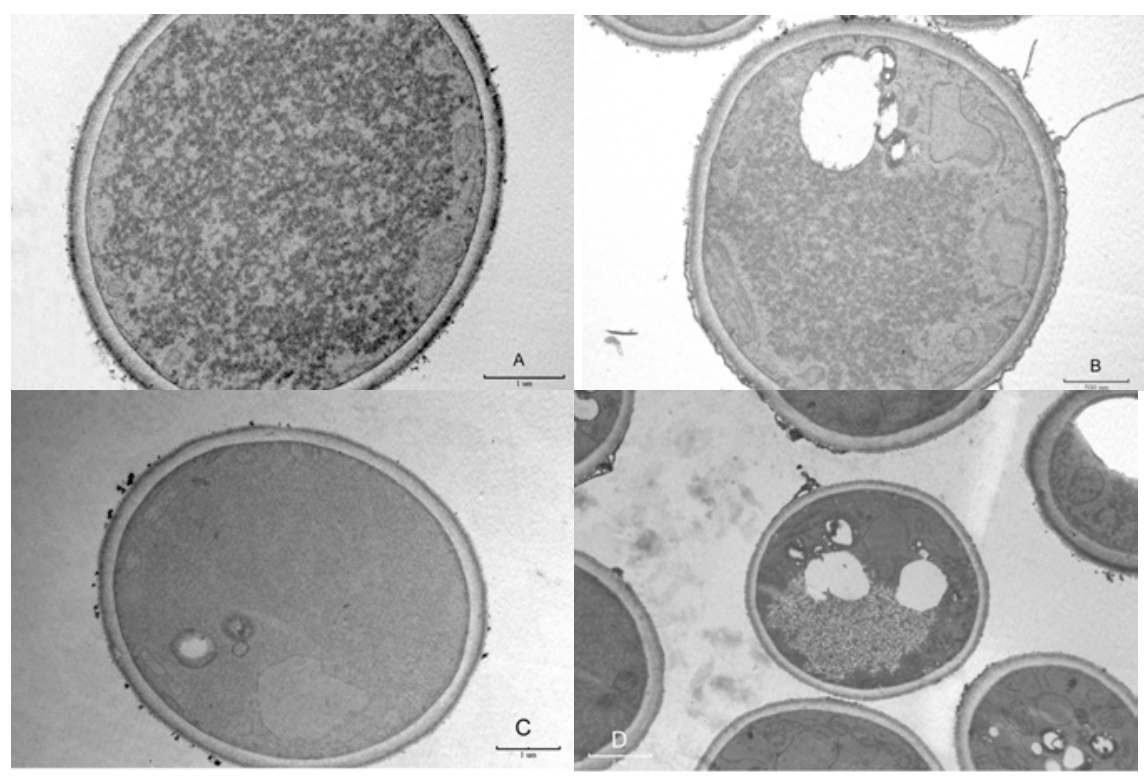

Figure 2. Morphology of C. albicans 36232. A. control cells are typically void of vacuoles and have a cytoplasm with abundant ribosomes. B. DBP-treated cells contain noticeably more vacuoles than control cells and exhibit cell wall peeling. C. In the Tween 80-treated cells, the most noticeable difference was an increase in the thickness of the cell wall. D. For the DBPT-treated cells, there is increased vacuole content, some cell wall peeling and a thicker cell wall. 